\title{
Social cohesion, structural holes, and a tale of two measures
}

\author{
V. Latora, ${ }^{1}$ V. Nicosia, ${ }^{1}$ and P. Panzarasa ${ }^{2}$ \\ ${ }^{1}$ School of Mathematical Sciences, Queen Mary University of London, E1 4NS London (UK). \\ ${ }^{2}$ School of Business and Management, Queen Mary University of London, E1 4NS London (UK)
}

\begin{abstract}
In the social sciences, the debate over the structural foundations of social capital has long vacillated between two positions on the relative benefits associated with two types of social structures: closed structures, rich in third-party relationships, and open structures, rich in structural holes and brokerage opportunities. In this paper, we engage with this debate by focusing on the measures typically used for formalising the two conceptions of social capital: clustering and effective size. We show that these two measures are simply two sides of the same coin, as they can be expressed one in terms of the other through a simple functional relation. Building on this relation, we then attempt to reconcile closed and open structures by proposing a new measure, Simmelian brokerage, that captures opportunities of brokerage between otherwise disconnected cohesive groups of contacts. Implications of our findings for research on social capital and complex networks are discussed.
\end{abstract}

PACS numbers: 89.75.Hc, 89.65.Ef, 87.23.Ge, 89.75.-k

Keywords: Social networks, Social capital, Social cohesion, Structural holes, Clustering, Effective size, Simmelian brokerage

\section{INTRODUCTION}

A fundamental idea in the social sciences is that social capital originates from social relations. As a result, social structure has long been seen as playing a crucial role in sustaining or hindering a wide range of performancerelated outcomes, both at the individual and collective levels 26, 27, 40 42. However, while social scientists tend to agree on the salience of social structure, there is still controversy over which type of social structure matters as a source of social capital. Over the years, scholars have typically advocated two opposite types of structure: the "closed" and "open" structures. On the one hand, proponents of the benefits of closed structures draw on the idea that social cohesion fosters trust [12, 15, 53, 63] and a sense of belonging 14], sustains cooperative behaviour 14, 33] and the enforcement of social norms [14, 24, 27], and facilitates the creation of a common culture [45]. On the other, advocates of the benefits of open structures emphasise the value that actors can extract from being located near structural holes separating non-redundant contacts, and thus from acting as brokers between otherwise disconnected others 7, 9, 10, 42, 60].

This paper aims to draw on the interplay between these two alternative conceptions of social capital, and engage with the ongoing debate over the relative benefits associated with closed and open structures. The relation between these two types of social structure will be explored through a comparative analysis of the measures with which these structures have traditionally been operationalised and formalised: the clustering coefficient and the effective size of an actor's local neighbourhood. In particular, we will show that it is possible to derive a simple mathematical relation between the clustering coefficient and the effective network size of a node. The existence of this relation between the two measures, which have originally and independently been introduced with the purpose of formalising two different concepts, supports the idea that social cohesion and structural holes are no more than the two sides of the same coin. Both measures can indeed be expressed in terms of number of links and number of triangles incident upon a node.

The question as to whether social capital stems from open or closed structures will probably always remain a matter of debate. There are cases where one is more interested in the benefits coming from closed structures 24], and other cases where, conversely, it is more convenient to exploit the existence of open structures 60. However, our work highlights that, to characterise the local structural properties of a node, it is equally informative to measure the clustering coefficient or the effective size of the node's local neighbourhood. Drawing on this, we will then use the relation between clustering and effective size to develop a novel measure for a generative mechanism of social capital that lies at the interface between closed and open structures: Simmelian brokerage. Being sensitive to variations in the position of links across local networks of the same density, this measure can capture opportunities of brokerage between otherwise disconnected groups of densely interconnected nodes [19, 34 36, 61, 67]. For this reason, Simmelian brokerage can be seen as suitable for formalising structures that lie at the interface between the closed and open ones [9, 61, 67]: it captures the extent to which a node's local network is characterised, on the one hand, by a combination of structural cleavages between distinct groups of contacts, and on the other by a closed cohesive structure within the boundaries of each group of contacts.

The paper is organised as follows. In Section ${ }_{\square}$ we introduce the concept of social capital, and offer a general overview of the two main theoretical conceptions of its structural foundations. In Section n we review the definitions of clustering coefficient and network effect- 
ive size. In Section we show that the two measures are linked through a simple mathematical relation, based on which, in Section o we introduce our new measure of Simmelian brokerage. Finally, in Section 口we extend the relation between clustering and effective size to the case of weighted graphs, and sketch out a definition of weighted Simmelian brokerage. The last Section will summarise and discuss our main findings.

\section{STRUCTURAL FOUNDATIONS OF SOCIAL CAPITAL}

The premise that seems to underpin most perspectives on social capital is the idea that investments in social relations yield expected returns in the marketplace, including the community, the economic, financial, political, and labour markets [40, 41]. As argued by Coleman, social capital can be characterised by two distinct properties: it "inheres in the structure of relations between actors and among actors", and "like other forms of capital, [it] is productive, making possible the achievement of certain ends that in its absence would not be possible" 14, p.S98].

Social scientists have long agreed on the salience of social structure as a source of social capital [26, 27, 40 42]. For instance, social structure has been seen as playing a pivotal role in sustaining individuals' and organisations' efforts to create value 24, 33, 44, 54, generate and transfer new knowledge [13, 28, 53, 61, 62], and enhance creativity $1,66,20,46,50,59,64]$. As suggested by a number of scholars 27, 40], three main explanations can be offered as to why social structure affects the outcomes of purposive actions. First, social structure can facilitate or hinder the flow of information, and in so doing it also impacts on its quality [28, 53, 62, 64]. Second, social structure can be seen as a source of reward and punishment due to the effects that social relations have on the internalisation and enforcement of social norms, including those against free-riding 25, 33]. Third, social structure nurtures and promotes the attainment of actors' trust, reputation, social credentials, status, identity and recognition through processes of third-party referrals and reinforcement of interactions [40, 41, 63].

Despite the convergence on the explanatory relevance of social structure, however, there is still controversy and debate over the type of social structure that matters as a source of social capital [2, 4, 9, 23, 40, 41, 54]. Theoretical conceptions of the structural foundations of social capital vacillate between two positions that vary in their understandings of the benefits associated with two opposite types of social structure: "closed" and "open" structures. Arguments in favour of each of these structures have been inspired by distinct rich traditions in sociological theory. Both arguments, however, are conceptually rooted in Simmel's seminal theoretical contributions on the expansion of a dyadic relationship into a three-party relationship ("Verbindung zu dreien"), and the sociological significance of the third element [58]. Simmel argued that the introduction of a third party fundamentally changes the social dynamics of a dyadic tie: "The appearance of the third party indicates transition, conciliation, and abandonment of absolute contrast (although, on occasion, it introduces contrast)." [58, p.145]. Simmel's emphasis here is on the two alternative functional roles the third party can play in the triad: the "non-partisan" or mediator with the tertius iungens (or "the third who joins") orientation on the one hand [46], and the broker with the tertius gaudens (or "the third who enjoys") orientation on the other [7].

\section{Closed structures and social cohesion}

Proponents of the benefits of closed structures typically build on Simmel's [58] tertius iungens logic and Coleman's [14, 15] conception of social capital predicated on the mechanism of social cohesion 22]. Over the years, the Simmelian triad has provided the theoretical backdrop against which scholars have investigated the relational hypothesis that actors separated by one intermediary are more likely to become connected with each other than actors that do not share any common acquaintance [17, 18, 29, 30, 43, 68, 69]. At the macro level of a social system, the tendency of actors to forge links locally within groups is conducive toward the creation of cohesive social structures, organised into well-defined tightly knit communities that are densely connected within but not across boundaries 21, 37.

One of the most influential theories of social capital, advocated by Coleman [14], is predicated precisely on the benefits that actors accrue from being socially embedded within cohesive social structures, rich in thirdparty relationships. Among the closure-based sources of social capital are normative control and deviance avoidance [9, 27, 40, 41]. More generally, network closure enables the emergence and enforcement of social norms by encouraging the internalisation of standards of acceptable behaviour and facilitating the detection and punishment of defective behaviour [33, 63]. In addition, it has been documented that being part of a close-knit group engenders a sense of belonging [14], fosters trust [12, 15, 53, 63], facilitates the exchange of fine-grained, complex, tacit, and proprietary information [28, 63], enables the creation of a common culture and the emergence of a shared identity 45], and helps sustain a high level of cooperation [14, 33].

Despite the benefits associated with social cohesion, the tendency of individuals to cluster into densely connected communities also bears a two-fold cost: local redundancy and social pressure. On the one hand, the more an actor's contacts are connected with each other, 
the less likely they are to take the actor closer to diverse sources of knowledge and resources that the actor is not already able to access [26]. Paucity of connections with new and non-redundant social circles may create isolation and eventually degrade social capital. This is the central argument of Burt's 7] seminal contribution on the benefits associated with occupying brokerage positions between otherwise disconnected individuals or groups in a network. On the other hand, above and beyond the redundancy of knowledge and resources, a cohesive structure can still exert a negative influence on the connected actors as a result of the social pressure favouring convergent thinking and group consensus. As dense third-party relationships engender reciprocal behaviour and sustain high degrees of similarity among the actors, they are conducive toward the maintenance of the status quo rather than the exploration of novel paths leading to divergent solutions [20, 59].

\section{Open structures and brokerage}

Both types of costs - redundancy and social pressure associated with social cohesion have inspired an alternative conception of social capital, typically distilled into the proposition that there are benefits actors can extract from participating in open structures that are rich in cleavages and opportunities of brokerage [7, 9, 10, 42, 60]. At the heart of this conception of social capital lies Simmel's 58] characterisation of the role of tertius gaudens in a triad. While the non-partisan tertius iungens aims "to save the group unity from the danger of splitting up" [58, p.154], the tertius gaudens wishes to create or intensify discontinuities in the social structure by forging or preserving unique ties to disconnected others.

The idea that social capital can originate from brokerage opportunities associated with structural gaps has been explored most thoroughly by Burt, who has perhaps contributed more than any other sociologist in recent decades to examine the structural features and performance implications of brokerage, especially in organisational domains $7-10]$. Burt defines a structural hole as the "separation between non-redundant contacts", "a relationship of non-redundancy between two contacts", "a buffer" that enables the two contacts to "provide network benefits that are in some degree additive rather than overlapping" [7, p.18]. Burt further identifies two sources of the social capital that an actor can mobilise by acting as the broker between contacts at the opposite sides of the hole: information benefits and control benefits. On the one hand, information benefits originate from the fact that, in open structures rich in structural holes, connections tend to be weak [26] and are likely to link people with different ideas, interests and perspectives [8]. By gaining exposure to a greater variance and novelty of information, actors embedded in brokered structures will be creative and successful in their endeavours [8, 20, 59]. On the other, control benefits are related to the third party's ability to gain an advantage by negotiating his or her relationships with disconnected others and turning their "forces combined against him into action against one another." [58, p. 162]. Preserving and fostering disunion between parties thus enable the actor standing near a structural hole to extract social capital buried in the hole, by playing the disconnected parties' demands and preferences against one another.

\section{The trade-off between closed and open structures}

A number of empirical studies have attempted to reconcile the two positions on social capital, and provide an integrative account of social cohesion and brokerage [2, 20, 50, 55, 61, 67]. Even though the routes pursued to develop a unified conception of social capital vary both theoretically and methodologically, scholars seem to converge on the idea that the benefits originating from social structure are contingent on a number of social, structural, and environmental conditions [2, 20, 50, 55], and that a suitable combination of the two types of structure can outperform each individual type in isolation [54, 61, 67].

A substantial body of the literature has examined the trade-off between social cohesion and brokerage by focusing on the interplay between social structure and the attributes of the interacting individuals, and suggesting that the benefits of either type of structure - closed or open - are contingent upon such attributes 50, 54, 55. In this vein, for example, Fleming et al. 20] have empirically examined the mitigating effects exerted by individuals' attributes on the benefits associated with brokerage. Their study suggests that, while brokerage between otherwise disconnected collaborators makes all individuals more likely to create new ideas, at the same time there are marginal contingent positive effects of social cohesion on generative creativity when individuals and their collaborators bring broad experience, have worked for multiple organisations, and have connections with external contacts. Similarly, Perry-Smith 50] has offered evidence suggesting that connections to contacts with heterogeneous background mediate the relationship between weak ties and creativity, and that there are interaction effects between centrality and number of outside ties upon creativity.

Another related line of investigation has suggested that an appropriate combination of cohesion and brokerage opportunities can provide individuals with the necessary redundant relationships as well as access to nonredundant information that facilitate task execution and enhance performance [9, 51, 54]. In this vein, there have recently been attempts to address and resolve the tradeoff between closed and open structures by advocating a conception of social capital that is contingent on the 
microstructural context in which bridging ties are embedded. From this perspective, Tortoriello and Krackhardt 61] have argued that brokerage is most beneficial when the the bridging tie is a Simmelian one, namely a tie in which the parties involved are reciprocally and strongly connected to each other as well as reciprocally and strongly connected to at least one common third party [34, p. 24]. Because the advantages traditionally associated with open structures have been found to be contingent upon the Simmelian nature of the bridging ties, this study has provided empirical evidence in favour of an integrative account of social capital, according to which individuals can extract benefits not simply from structural holes or a cohesive neighbourhood in isolation, but from a combination of both structural configurations.

More recent work has proposed a refined contingent conception of social capital by recasting the trade-off between closed and open structures in terms of the tradeoff between "channel bandwidth" (i.e., tie strength) and "network diversity" (i.e., richness in structural holes) [2]. The main argument is that, while structural gaps remain sources of diverse information, the total amount of useful novel information tends nonetheless to be positively affected also by how strongly and frequently individuals interact with one another. Strong relationships, characterised by frequent social interactions, typically found in cohesive closed structures [7, 26], are likely to sustain the flow of a large volume of rich non-redundant information that, it is claimed, "tends to be more detailed, cover more topics, and address more complex, interdependent concepts" [2, p. 94] than the information flowing in a network rich in weak ties with less frequent interactions. However, because the strength of ties tends to contract as the social structure becomes richer in cleavages and brokerage opportunities, and thus more diverse [7, 26], then a trade-off exists between network diversity and tie strength as they produce counterbalancing effects on the access to novel information. This trade-off is resolved by regarding the relative benefits of network diversity and tie strength as contingent on the social settings and information environments in which individuals interact. In particular, evidence has suggested that tie strength (i.e., closed structures) trumps network diversity (i.e., open structures) as the topic space becomes broader, information is frequently updated, and the overlap between the information possessed by an individual's contacts becomes larger [2].

In this paper, we draw on these recent studies on social capital, and contribute to the ongoing debate in a two-fold way. First, we formalise the trade-off between closed and open structures by proposing a functional relation between the measures with which these two types of structure have traditionally been operationalised. Unlike other studies [e.g., 2, [20, 61], we do not carry out an empirical investigation to test the relative advantages of different structural configurations. By contrast, we offer a rigorous and quantitative framework for a better understanding of the trade-off between two concepts - cohesion and structural holes - that have heretofore been compared to each other primarily, if not exclusively, on intuitive grounds.

Our second contribution to the debate builds on the proposed formalisation of the relationship between cohesion and structural holes to offer a new measure Simmelian brokerage - for detecting the degree to which an individual's structural position lies at the interface between a closed and an open structure. This measure is inspired by, and is in qualitative agreement with, other studies that have suggested the idea that social capital can originate simultaneously from both social cohesion and structural holes [54, 61, 67]. However, it differs from previous formalisations in two ways. First, unlike other studies [67], we do not use clique percolation methods [49] to uncover an overlapping community structure and construct a group-level measure of group intersection and multiple membership. Second, unlike other scholars, we do no rely upon actor-level attributes (e.g., tenure) 54 or exogenously defined cross-boundary relationships [61] to detect network heterogeneity and structural gaps in an individual's local neighbourhood. By contrast, the novelty of our measure lies precisely in the fact that it is defined at the node level and detects directly, based on the node's local neighbourhood, the extent to which the node belongs to multiple groups that are both tightly knit and disconnected from each other. In this sense, Simmelian brokerage dovetails with the idea that multiple group membership enables a node to extract social capital from its underlying structure by blending social cohesion with structural holes.

\section{MEASURING SOCIAL COHESION AND STRUCTURAL HOLES}

If social cohesion and structural holes have long represented two distinct conceptual pillars, each underlying one of the two opposing conceptions of social capital, they have also been formalised through two distinct, and independently developed, measures: respectively, the clustering coefficient and the effective size of a node's local network. While clustering has typically been used for measuring the extent to which a node is socially embedded within a closed cohesive structure [17, 29, 43, 69], effective size is a measure for detecting the non-redundancy of a node's contacts, and therefore the degree to which the node's local neighbourhood is rich in structural holes [7]. The remaining of this Section is organised into two parts. Section $_{\mathbf{a}}$ will review the definition of node clustering coefficient, and discuss an alternative measure for cohesion, node local efficiency. Section „ will be devoted to the formalisation of effective size. 


\section{Clustering coefficient and local efficiency}

Let us consider an unweighted undirected graph $G(V, L)$ with $N=|V|$ nodes and $K=|L|$ links, and let us focus on one of its nodes, node $i$, with $i \in\{1,2, \ldots, N\}$. In order to measure the local cohesion of node $i$, we define the subgraph $G_{i}$ induced in $G$ by the set $\mathcal{N}_{i}$ of the neighbours of $i$. The node clustering coefficient $C_{i}$ of node $i$ can then be defined as [68, 69]:

$$
C_{i}=\left\{\begin{array}{cc}
\frac{K\left[G_{i}\right]}{k_{i}\left(k_{i}-1\right) / 2} & \text { for } k_{i} \geq 2 \\
0 & \text { for } k_{i}=0,1
\end{array}\right.
$$

where $K\left[G_{i}\right]$ is the number of links in $G_{i}$. The node clustering coefficient $C_{i}$ indicates the probability that two neighbours of node $i$ are connected by a link, and is properly normalised by definition such that $0 \leq C_{i} \leq 1$. In fact, the value of $C_{i}$ in Equation (11) is the ratio between the actual number of links $K\left[G_{i}\right]$ in the subgraph induced by the first neighbours of $i$ and their maximum possible number, that is $\left(\begin{array}{c}k_{i} \\ 2\end{array}\right)=k_{i}\left(k_{i}-1\right) / 2$. Notice that $K\left[G_{i}\right]$ is also equal to the actual number of triangles containing node $i$, while $k_{i}\left(k_{i}-1\right) / 2$ is the number of open triads centred on $i$, which corresponds to the maximum possible number of triangles containing a node $i$ with $k_{i}$ links. Therefore, the node clustering coefficient $C_{i}$ in Equation (1) can be alternatively seen as the proportion of triads centred in $i$ that close into triangles.

As an example, let us consider the two graphs $G^{a}$ and $G^{b}$ shown in Figure 1. In both graphs, node $i$, coloured in yellow, has degree $k_{i}=4$, so that the sets $\mathcal{N}_{i}^{a}$ and $\mathcal{N}_{i}^{b}$ of the neighbours of $i$ contain four nodes each. The four neighbours of $i$, labelled as nodes $1,2,3,4$, are shown as red circles, while the links connecting these nodes to $i$ are shown as dashed lines. The subgraph $G_{i}^{a}$ induced in $G^{a}$ by the set $\mathcal{N}_{i}^{a}$ of the neighbours of $i$ has four nodes and three links shown as solid lines. Similarly, the subgraph $G_{i}^{b}$ induced in $G^{b}$ by the set $\mathcal{N}_{i}^{b}$ contains four nodes and three links. Therefore, the clustering coefficient of node $i$ is equal to $1 / 2$ in both cases. In fact, the four nodes of the induced graphs can be connected to each other through at most six links. In the figure, only three of these potential links are present. Hence, $C_{i}=3 / 6=$ $1 / 2$. Notice that, in general, the subgraph $G_{i}$ of the neighbours of node $i$ can be unconnected. For instance, this happens for the case of graph b) in Figure 1] where node 4 is isolated in subgraph $G_{i}^{b}$. However, this does not affect the mathematical definition of the clustering coefficient $C_{i}$. What is instead problematic is a node $i$ which is itself an isolate in the graph, or has only one link. In this case, the ratio $2 K\left[G_{i}\right] /\left(k_{i}\left(k_{i}-1\right)\right)$ is not defined. The usual convention, in this case, is to set $C_{i}=0$ when the degree of $i$ is either zero or one, as reported in Equation (11).
An important feature of the clustering coefficient of node $i$, as defined in Equation (1), is that it only depends on the number of links in the subgraph $G_{i}$, and not on which pairs of nodes are actually connected through such links in $G_{i}$. Notice, for instance, that both $G_{i}^{a}$ and $G_{i}^{b}$ in Figure 1 have three links, but while $G_{i}^{a}$ is a line of four nodes, $G_{i}^{b}$ consists of a triangle and an isolated node. For this reason, here we discuss an alternative measure for the cohesion of the neighbourhood of a node, the node local efficiency, that enables the two cases shown in Figure 1 to be clearly differentiated.

In an unweighted undirected graph $G(V, L)$, the node local efficiency of node $i$ is defined as the efficiency of the subgraph $G_{i}$ [38, 39], where the efficiency of a graph is the average of the inverse of the distances between the nodes of the graph. Therefore, the node local efficiency, $E_{i}$, of node $i$ can be written as:

$$
\begin{aligned}
& E_{i}=E\left[G_{i}\right]=\frac{1}{k_{i}\left(k_{i}-1\right)} \sum_{\ell \in \mathcal{N}_{i}} \sum_{\substack{m \in \mathcal{N}_{i} \\
m \neq \ell}} \epsilon_{\ell m}= \\
& =\frac{1}{k_{i}\left(k_{i}-1\right)} \sum_{\ell \in \mathcal{N}_{i}} \sum_{\substack{m \in \mathcal{N}_{i} \\
m \neq \ell}} \frac{1}{d_{\ell m}}
\end{aligned}
$$

where $E\left[G_{i}\right]$ stands for the efficiency of graph $G_{i}$, while $\epsilon_{\ell m}$ measures the reachability between node $\ell$ and node $m$, and is set equal to the inverse of the distance $d_{\ell m}$ between the two nodes. Notice that distances between nodes are evaluated on the graph $G_{i}$, and not on graph $G$. Moreover, the local efficiency is properly normalised by definition, such that $0 \leq E_{i} \leq 1$. Therefore, it takes values in the same range as the clustering coefficient.

By making use of Equation (2), it is possible to distinguish between the roles that node $i$ plays in the two graphs $G^{a}$ and $G^{b}$ in Figure 1. If we calculate the distances between the four neighbours of $i$ in $G_{i}^{a}$, we obtain: $d_{12}=d_{23}=d_{34}=1, d_{13}=d_{24}=2$ and $d_{14}=3$. Hence, we have $\epsilon_{12}=\epsilon_{23}=\epsilon_{34}=1, \epsilon_{13}=\epsilon_{24}=1 / 2$, and $\epsilon_{14}=1 / 3$, so that the local efficiency of node $i$ in graph a) of Figure 1 is $E_{i}^{a}=13 / 18$. Conversely, if we consider $G_{i}^{b}$, we obtain: $d_{12}=d_{23}=d_{13}=1, d_{14}=d_{24}=d_{34}=\infty$. Thus, we have: $\epsilon_{12}=\epsilon_{23}=\epsilon_{13}=1, \epsilon_{14}=\epsilon_{24}=\epsilon_{34}=0$, and in this case the local efficiency of node $i$ is $E_{i}^{b}=1 / 2$, which is smaller than $E_{i}^{a}$. Thus, even if node $i$ has the same clustering coefficient in the two graphs, its local efficiency is different in the two cases.

The mathematical definition of efficiency we have adopted implies that the efficiency of a graph with a fixed number of nodes becomes larger as the number of links increases. And for graphs with the same number of nodes and the same number of links, the efficiency depends on where the links are actually located in each graph. In particular, the efficiency of a chain of three links connecting four nodes is higher than the efficiency of a triangle combined with an isolated node. The reason for this is that, in the latter case, the presence of an isolated node affects 


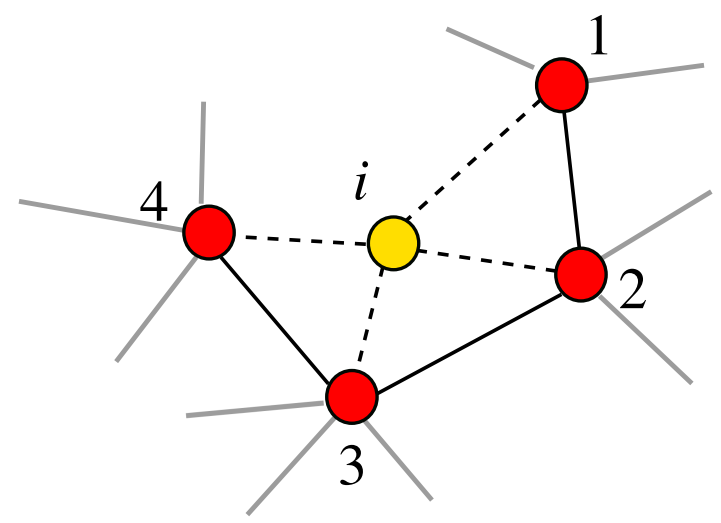

a)

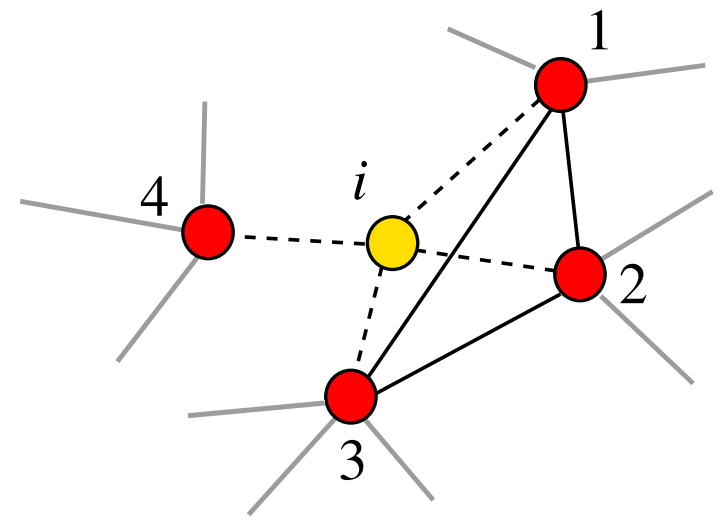

b)

Figure 1. The clustering coefficient of a node, as defined in Ref. [69], measures the cohesiveness of the node's neighbourhood. Despite the different configurations of links, the clustering coefficient of node $i$, measured as the fraction of links among $i$ 's neighbours over the total possible number of such links, is equal to $C_{i}=1 / 2$ in both graphs. Conversely, the local efficiency of node $i$ in graph a) is larger than in graph b).

the overall reachability among nodes in the system: notwithstanding the presence of a triad of connected nodes, the isolated node is not reachable from the other ones.

\section{Effective size}

The original formalisation of the idea of structural holes was proposed by Burt for weighted graphs [7]. Therefore, here we will first begin our analysis of measures for open structures with the most general case of a directed weighted graph $G(V, L, W)$. We will then restrict our focus to the particular case of undirected unweighted graphs, and return to the general case of weighted graphs in Section

Let us indicate as $w_{i j} \geq 0$ the $(i, j)$ entry of the weighted asymmetric matrix that describes the directed weighted graph $G(V, L, W)$. As argued by Granovetter [26], the weight of a link between any nodes $i$ and $j$ has the following meaning: a high (low) value of $w_{i j}$ indicates a large (small) amount of time, emotional intensity, intimacy, and reciprocal services that characterise the link connecting node $i$ to node $j$. Among the various measures introduced by Burt to detect and quantify the presence of structural holes, a key role is played by the effective size of a node's local network.

The effective size of node $i$ 's network indicates the extent to which each of the first neighbours of $i$ is redundant with respect to the other neighbours, and can be expressed in terms of the two following matrices: the transition matrix $P$ and the marginal strength matrix $M$. The entry $p_{i \ell}$ of matrix $P$ measures the proportion of $i$ 's network time and energy invested in the relationship with node $\ell$, and is defined as [7]:

$$
p_{i \ell}=\frac{w_{i \ell}+w_{\ell i}}{\sum_{m}\left(w_{i m}+w_{m i}\right)}
$$

where $w_{i \ell}+w_{\ell i}$ is the sum of the weights of the two links connecting $i$ to $\ell$, while $\sum_{m}\left(w_{i m}+w_{m i}\right)$ is the total strength of node $i$. This is the sum of the out-strength, $s_{i}^{\text {out }}=\sum_{m} w_{i m}$, and the in-strength, $s_{i}^{\text {in }}=\sum_{m} w_{m i}$, of $i$, i.e., the sum of the weights of all the incoming and outgoing links incident upon $i$. Notice that by definition $0 \leq p_{i \ell} \leq 1 \forall i, \ell$, with $p_{i \ell}=0$ if there is neither a link from $i$ to $\ell$, nor a link from $\ell$ to $i$. Also, the transition matrix $P$ is stochastic: $\sum_{\ell} p_{i \ell}=1$. The entry $m_{j \ell}$ of the second matrix, the marginal strength matrix $M$, is defined as:

$$
m_{j \ell}=\frac{w_{j \ell}+w_{\ell j}}{\max _{m}\left(w_{j m}+w_{m j}\right)}
$$

Again, $0 \leq m_{j \ell} \leq 1 \forall j, \ell$, with $m_{j \ell}=0$ if there is neither a link from $j$ to $\ell$, nor from $\ell$ to $j$. Notice that the two matrices $P$ and $M$ defined above are non-symmetric.

According to the definition given by Burt, the effective size $\mathcal{S}_{i}$ of node $i$ 's network reads [5, [7]:

$$
\mathcal{S}_{i}=\sum_{j \in \mathcal{N}_{i}}\left[1-\sum_{\ell} p_{i \ell} m_{j \ell}\right]
$$

where $\mathcal{N}_{i}$ is the set of neighbours of $i$. Excluding the case where $i$ is an isolate, for which $\mathcal{S}_{i} \equiv 0$ by definition, in general $1 \leq \mathcal{S}_{i} \leq k_{i} \forall i$, that is the effective size of node $i$ 's network ranges from its smallest value equal to 1 , when node $i$ belongs to a clique, to a maximum value equal to the node degree $k_{i}$, when there are no links $(j, \ell)$ connecting any two neighbours $j$ and $\ell$ within $i$ 's network, i.e. when $i$ is the centre of a star graph. In general, the 
more redundant the neighbours of $i$ are, the smaller the value of $\mathcal{S}_{i}$ is, and vice versa.

The expressions above largely simplify in the case in which the graph is undirected and/or unweighted. In fact, when the graph is undirected, we have: $w_{i \ell}=$ $w_{\ell i} \forall i, \ell$. In this case, the entries of the transition matrix $P$ and of the marginal strength matrix $M$ read, respectively:

$$
p_{i \ell}=\frac{w_{i \ell}}{\sum_{m} w_{i m}}=\frac{w_{i \ell}}{s_{i}^{\text {out }}}
$$

and

$$
m_{j \ell}=\frac{w_{j \ell}}{\max _{m} w_{j m}}
$$

Furthermore, if the graph is also unweighted, we have: $w_{i \ell}=a_{i \ell} \forall i, \ell$, where $a_{i \ell}$ is equal to 1 if there is a link between $i$ and $\ell$, and to zero otherwise. This implies that $\max _{m} w_{j m}=\max _{m} a_{j m}=1$ for any node $j$ that is not an isolated node. Consequently, the entries of the transition matrix $P$ and of the marginal strength matrix $M$ reduce to:

$$
p_{i \ell}=\frac{a_{i \ell}}{\sum_{m} a_{i m}}=\frac{a_{i \ell}}{k_{i}}
$$

and

$$
m_{j \ell}=a_{j \ell}
$$

In this case, $P$ is the transition probability of a random walk on the graph [16], while the marginal strength matrix $M$ coincides with the adjacency matrix $A$.

Let us now consider the following example to illustrate the meaning of Eq. (5). Figure 2 reports two undirected unweighted graphs, $G^{a}$ and $G^{b}$, having the same number of nodes, $N=8$, and the same number of links, $K=13$. In both graphs, node $i$ is coloured in yellow and has $k_{i}=5$ links to its neighbours, shown as dashed lines. The links connecting the neighbours of $i$ are indicated by solid black lines. By visual inspection it can be easily noticed that the neighbourhood of $i$ in graph $G^{b}$ contains more redundant links than in $G^{a}$. Indeed, if we evaluate the effective size of node $i$ in graph $G^{a}$, we find that the contribution of three out of the five neighbours in $i$ 's network towards the summation in Equation (5) is equal to 1. This is because each of these three nodes in $\mathcal{N}_{i}^{a}$ has no links to the other neighbours of $i$, and therefore is nonredundant. The contribution of each of the other two remaining neighbours in $i$ 's network towards the summation in Equation (5) is equal to $1-1 / k_{i}$. We finally have: $\mathcal{S}_{i}^{a}=1+1+1+(1-1 / 5)+(1-1 / 5)=3+8 / 5=23 / 5$, which is a number larger than 3 , but smaller than the actual degree $k_{i}=5$ of the node. If we calculate the effective size of node $i$ in graph $G^{b}$, we obtain: $\mathcal{S}_{i}^{b}=$ $(1-1 / 5)+(1-2 / 5)+(1-2 / 5)+(1-2 / 5)+(1-1 / 5)=17 / 5$. Because of the higher redundancy of the nodes in $\mathcal{N}_{i}^{b}$, this value is smaller than the effective size of node $i$ 's network in graph $G^{a}$.

\section{A SIMPLE RELATION BETWEEN CLUSTERING AND EFFECTIVE SIZE}

The two examples outlined in the previous Section already point to the existence of a relation between the clustering coefficient and the effective size of a node's network in an unweighted graph. In fact, the values of $C_{i}$ and $\mathcal{S}_{i}$ both depend on the number of triangles containing node $i$. The larger the number of triangles, i.e. of closed structures involving node $i$, the larger the clustering coefficient $C_{i}$. Conversely, the smaller the number of triangles, the larger the number of open structures that can be exploited by $i$, and thus the larger the effective size of node $i$ 's network. Therefore, we expect an inverse relation between $C_{i}$ and $\mathcal{S}_{i}$ : the larger the clustering coefficient of a node, the smaller the effective size of the node's network. In this Section, we show that there is indeed an exact and simple relation between the two measures. Based on this relation, it can then be argued that it is not necessary to operationalise two distinct concepts (cohesion and structural holes) and use two different measures (clustering and effective size) to investigate two distinct sources of social capital, when one source can be measured simply in terms of the other. More specifically, either measure, together with the degree of the node, is sufficient to quantify both the local cohesion and the structural holes characterising the node's local network.

We first notice that the definition of node clustering coefficient given in Equation (1) can be expressed in terms of the adjacency matrix of the graph. In fact, the number of links $K\left[G_{i}\right]$ in graph $G_{i}$ can be easily calculated from the adjacency matrix by observing that $\left(A^{3}\right)_{i j}=\sum_{\ell, m} a_{i \ell} a_{\ell m} a_{m j}$ is equal to the number of walks of length 3 connecting node $i$ to node $j$. In particular, by setting $i=j$, the quantity $\sum_{\ell, m} a_{i \ell} a_{\ell m} a_{m i}$ denotes the number of closed walks of length 3 from node $i$ to itself. This is twice the number of triangles containing node $i$. The generic triangle containing node $i$ and the two nodes $l$ and $m$ is made of the two links connected to node $i$, namely $(i, \ell)$ and $(m, i)$, and of the link $(\ell, m)$ that belongs to $G_{i}$. Since the link $(\ell, m)$ appears twice, namely in the closed walk $(i, \ell, m, i)$ and in the closed walk $(i, m, \ell, i)$, the number of links $K\left[G_{i}\right]$ is given by:

$$
K\left[G_{i}\right]=\frac{1}{2} \sum_{j, m} a_{i j} a_{j m} a_{m i} .
$$

Notice that this is the numerator of Equation (1), so that we can express the local clustering coefficient of node $i$ as:

$$
C_{i}=\left\{\begin{array}{cc}
\frac{\sum_{j, \ell} a_{i j} a_{j \ell} a_{\ell i}}{k_{i}\left(k_{i}-1\right)} & \text { for } k_{i} \geq 2 \\
0 & \text { for } k_{i}=0,1
\end{array}\right.
$$

Let us now consider the effective size of node $i$ 's network. When the graph is undirected and unweighted, 


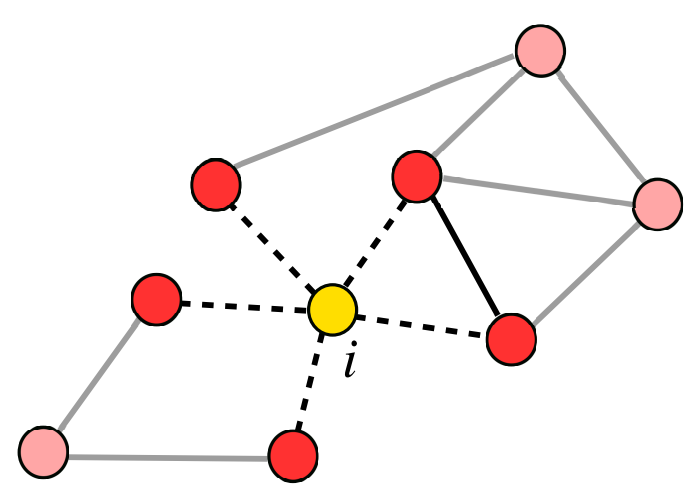

a)

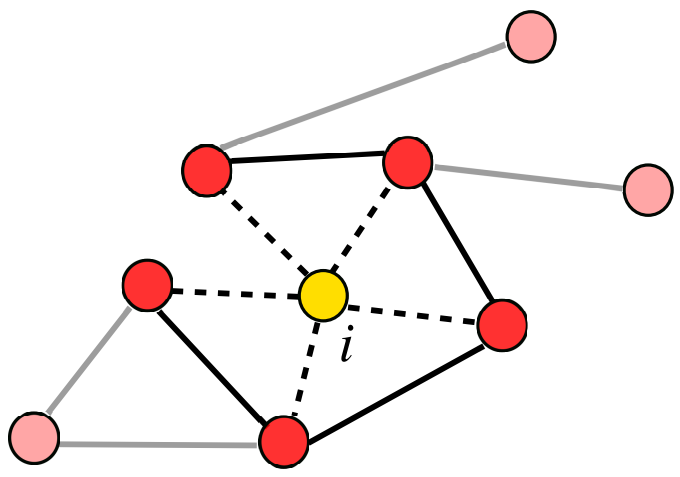

b)

Figure 2. The effective size of a node's network, as defined in Ref. 7], measures the lack of redundancy of the node's contacts. The neighbourhood of node $i$ in the first graph is less redundant than the one in the second graph. Consequently, the effective size, $\mathcal{S}_{i}^{a}=23 / 5=4.6$, of node $i$ in graph $G^{a}$ is larger than the effective size, $\mathcal{S}_{i}^{b}=17 / 5=3.4$, of node $i$ in graph $G^{b}$.

the entries of the transition matrix $P$ and of the marginal strength matrix $M$ read, respectively, as in Equations (8) and (9). Consequently, the effective size $\mathcal{S}_{i}$ of node $i$ 's network can be written as:

$$
\begin{aligned}
\mathcal{S}_{i} & =\sum_{j} a_{i j}\left[1-\sum_{\ell} p_{i \ell} m_{j \ell}\right]= \\
& =k_{i}-\sum_{j} a_{i j} \sum_{\ell} p_{i \ell} m_{j \ell}= \\
& =k_{i}-\sum_{j} \sum_{\ell} a_{i j} \frac{a_{i \ell}}{k_{i}} a_{j \ell}= \\
& =k_{i}-\frac{1}{k_{i}} \sum_{j} \sum_{\ell} a_{i j} a_{j \ell} a_{\ell i}
\end{aligned}
$$

If we plug the expression for the clustering coefficient of node $i$ in Equation (11) into Equation (12), we obtain:

$$
\mathcal{S}_{i}=k_{i}-\left(k_{i}-1\right) C_{i}
$$

This is an exact relation that connects three measures at the node level: effective size, clustering, and degree. For instance, we can use the relation to obtain the effective size of a node's network by measuring the clustering coefficient of the node. Since for the two graphs in Figure 2 we have $C_{i}^{a}=1 / 10$ and $C_{i}^{b}=4 / 10$, by using Equation 13 we obtain: $\mathcal{S}_{i}^{a}=5-4 / 10=23 / 5$ and $\mathcal{S}_{i}^{b}=5-16 / 10=17 / 5$, in perfect agreement with the values obtained by using Definition [5. More generally, Equation (13) provides a formalisation of the fact that structural holes and social cohesion are indeed the two faces of the same coin. The presence of structural holes, measured by the effective size of a node's network, depends only on the degree of the node and on the social cohesion of the node's local network, as measured by the node clustering coefficient. Conversely, the clustering coefficient of a node is uniquely determined by the degree of the node and by the effective size of its network.
By definition, the effective size of a node's network can take values between zero (if the node is an isolate) and the degree of the node, while the clustering coefficient varies from zero to one. To make the two quantities comparable, we can normalise the definition of effective size. To this end, we can define the normalised effective size of node $i$ 's network, $\mathcal{S}_{i}^{\prime}$, dividing the effective size $\mathcal{S}_{i}$ by its maximum possible value $k_{i}$, namely: $\mathcal{S}_{i}^{\prime}=\mathcal{S}_{i} / k_{i}$. If the node is an isolate, we set $\mathcal{S}_{i}^{\prime}=0$. When both measures are normalised in $[0,1]$, their relation reads:

$$
\mathcal{S}_{i}^{\prime}=1-\frac{k_{i}-1}{k_{i}} C_{i}
$$

which, for nodes with large degree, is well approximated by:

$$
\mathcal{S}_{i}^{\prime} \simeq 1-C_{i}
$$

This equation indicates that the clustering coefficient and the normalised effective size are indeed two complementary measures that can be defined one in terms of the other. As a result, this relation cautions against using both measures simultaneously for detecting sources of social capital. For instance, the inclusion of both clustering and effective size as covariates in a multivariate regression model would inevitably entail problems of multicollinearity due to the linear relation found between the two measures.

Drawing on this relation, we can express in terms of effective size many of the results obtained for the clustering coefficient in real networks. In particular, it has been found that in many networks the clustering coefficient of a node scales with the degree of the node as $k^{-\omega}$, where $0 \leq \omega \leq 1$ [52, 65]. This means that high-degree nodes tend to have a relatively smaller clustering coefficient than low-degree nodes. Consequently, in such networks effective size will increase with $k$, so that higher-degree 
nodes will exhibit a higher effective size than lower-degree nodes. The reason for this is precisely the mirror image of the argument typically proposed to explain the inverse relation between clustering and degree: any two neighbours of a large-degree node are more likely not to be directly connected with each other than any two neighbours of a low-degree node. Moreover, it is possible to use any model of random networks with a tunable degree-dependent clustering coefficient [31, 52, 57, 66] to construct a random network with a fixed distribution of effective size. In turn, such random network can be used as a null model to assess the statistical significance of the correlation, measured at the node level, between effective size on the one hand, and other structural properties or performance-related outcomes, on the other.

\section{RECONCILING SOCIAL COHESION AND STRUCTURAL HOLES: SIMMELIAN BROKERAGE}

As discussed in Section , local efficiency is a generalisation of the node clustering coefficient, in that it measures the extent to which the neighbours of node $i$ would reach each other if $i$ were removed from the network. Unlike the clustering coefficient, local efficiency allows us to distinguish between cases where the subgraphs $G_{i}$ have the same number of nodes and the same number of links, but are topologically different, as was the case of the two examples in Figure 1. In this Section, we show that local efficiency can be employed effectively to develop a new measure for brokerage that lies at the interface between clustering and effective size. Like clustering and effective size, this measure will be sensitive to structural gaps in a node's local network. However, unlike clustering and effective size, it will also be sensitive to variations in the position of links across local networks of the same density. For this reason, the measure is capable of capturing brokerage opportunities among otherwise disconnected socially cohesive groups of nodes.

We begin by observing that, if the local efficiency of node $i$ is small, then $i$ plays an important role in enabling and facilitating reachability among its neighbours. In this case, $i$ acts as a broker among its neighbours, since the removal of $i$ would disconnect many pairs of nodes in $i$ 's neighbourhood, or would inevitably deteriorate the ability of these nodes to reach each other. Conversely, if the local efficiency of $i$ is large, then the nodes in $i$ 's neighbourhood would still be able to reach each other even without the intermediary role of $i$, and as a result they would barely be affected by the removal of $i$. In this case, $i$ plays a negligible brokerage role. More generally, the higher the local efficiency of a node, the fewer the opportunities a node has to act as a broker, and vice versa. Based on this observation, on the relation in Equation (13), and on the fact that the clustering coefficient and the normalised effective size range in the same interval $[0,1]$, here we introduce the following measure for local brokerage $\mathcal{B}_{i}$ of node $i$ :

$$
\mathcal{B}_{i}=k_{i}-\left(k_{i}-1\right) E_{i}
$$

In qualitative agreement with Krackhardt's 34 36 idea of Simmelian ties as ties embedded in cliques, we propose to call this measure Simmelian brokerage. Our choice is motivated by the fact that the measure is indeed sensitive to the extent to which a node acts as a broker between Simmelian ties or, alternatively, between otherwise disconnected groups of densely connected nodes. This is the case of a node that is a member of different cliques, and thus acts as the intermediary between two or more disconnected sets of Simmelian ties, rich in third-party relationships [11, 19, 61, 67]. The definition of Simmelian brokerage is similar to that of effective size, with the only difference that the clustering coefficient $C_{i}$ of node $i$ is replaced by the node local efficiency $E_{i}$. According to Equation (16), when the degree of a node is fixed, an increase in the value of local efficiency corresponds to a decrease in the value of Simmelian brokerage, and vice versa.

To shed light on the relation between effective size and Simmelian brokerage, we now discuss a number of examples of brokerage opportunities in unweighted undirected graphs. Figure 3 shows six graphs, each with $N=9$ nodes, but with a different number and configuration of links. A first inspection of the figure makes it immediately clear that the central node $i$, indicated in yellow, has a different brokerage role in each of the six graphs. Graph a) is a clique, i.e. a complete graph in which each node has a link to each of the other nodes. This structure is characterised by high redundancy - indeed the maximum possible redundancy among all the graphs with the same number of nodes - due to the presence of the maximum possible number of links in the graph. In this case, the local efficiency of node $i$ is equal to $E_{1}^{a}=1.0$, and for Simmelian brokerage we obtain the smallest possible value, $\mathcal{B}_{i}^{a}=8-7 \times 1.0=1.0$. Such a small value is consistent with the relatively negligible role that $i$ has in facilitating reachability among its neighbours: if $i$ were removed from the graph, not only would its neighbourhood remain connected, but each of its contacts would still have a direct connection with each other. For the overall network, node $i$ is thus a redundant contact.

Graph b) is a wheel graph, where the neighbours of node $i$ are arranged in a cycle. As in the case of a clique, here the neighbourhood of node $i$ remains connected even when $i$ is removed from the graph, so that $i$ can be considered somehow redundant. However, while some pairs of nodes in the induced graph have distance equal to 1 , the majority of pairs of nodes are at distance 2,3 or 4 , so that the corresponding local efficiency is $E_{i}^{b} \simeq 0.559$ and node $i$ 's Simmelian brokerage is equal to $\mathcal{B}_{i}^{b} \simeq 4.083$. The relatively higher value of $i$ 's Simmelian brokerage in 


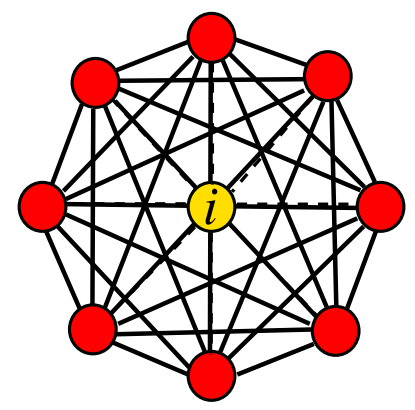

a)

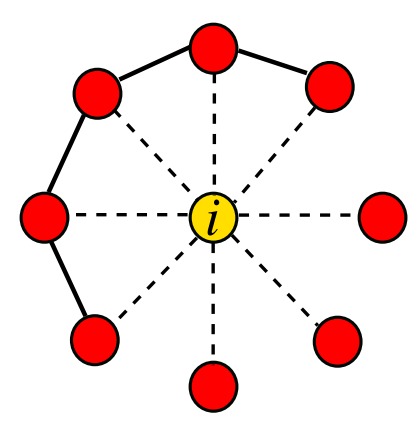

d)

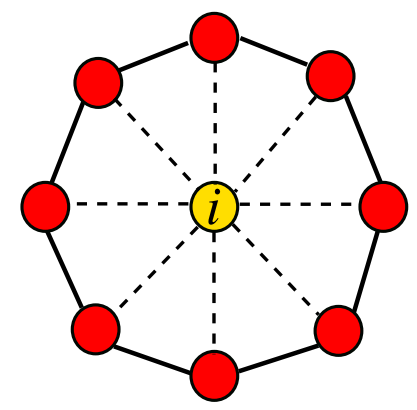

b)

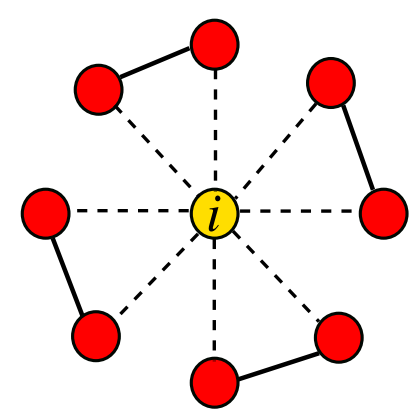

e)

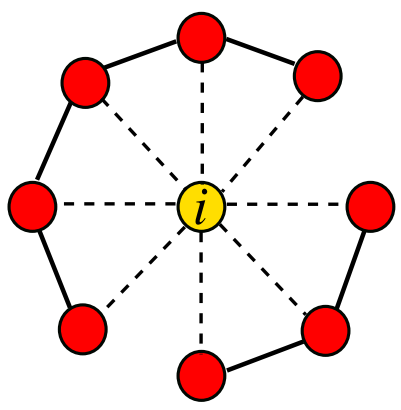

c)

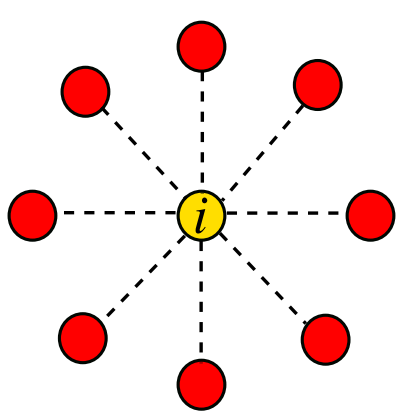

f)

Figure 3. The extent to which a node acts as a Simmelian broker depends on the number and configuration of links in its neighbourhood. The six graphs correspond to six values of $\mathcal{B}_{i}$, in increasing order: a) 1.0, b) 4.08, c) 5.83 d) 6.46 , e) 7.0 , f) 8.0. When the neighbourhood of $i$ is a clique (graph a)), the Simmelian brokerage of $i$ is negligible, and $i$ is practically superfluous. As soon as the neighbourhood becomes sparser and more structural holes appear, node $i$ acquires higher values of Simmelian brokerage. In graphs d) and e), node $i$ has the same clustering coefficient (and thus the same effective size), but the value of Simmelian brokerage of $i$ is higher in e) than in d) because in e) $i$ intermediates among four cohesive groups of nodes, whereas in d) $i$ intermediates between one group of four loosely connected nodes and three otherwise isolated nodes. Opportunities of brokerage are maximised in the limiting case of a star graph in which node $i$ intermediates among eight otherwise disconnected contacts (graph f)).

graph b) than in graph a) reflects the more central role played by $i$ in b) than in a) in facilitating reachability among its neighbours. This is also reinforced by the fact that, when $i$ is removed from the graph, the average distance among its neighbours increases from $\sim 1.714$ (in graph a)) to $\sim 2.286($ in graph b)).

From a visual inspection, we would also expect the Simmelian brokerage of node $i$ to increase from graph b) to graph c) due to an increase in structural gaps between distinct groups of nodes. In graph c), node $i$ does not only intermediate between already connected nodes, but it brokers between different groups of nodes that would otherwise remain disconnected. In this case, the local efficiency of $i$ is $E_{i}^{c} \simeq 0.309$ and, as expected, the corresponding Simmelian brokerage of $i$ is higher than in graph b), and is equal to $\mathcal{B}_{i}^{c} \simeq 5.83$.

Graphs d) and e) have the same number of links $K=$ 4 , but with a different configuration. Interestingly, the effective size of node $i$ 's network in the two graphs is the same, $\left(S_{i}=7.0\right)$, since in both graphs the clustering coefficient of $i$ is equal to $C_{i}=1 / 7$. However, a removal of $i$ causes a more significant damage in graph e) than in graph d), since the intermediary role of $i$ is more crucial in e) than in d). Moreover, in graph e) node $i$ is affiliated with four distinct cohesive groups of connected contacts, whereas in graph d) node $i$ intermediates between three isolated contacts and one loosely connected group. In this sense, in graph e) node $i$ spans more structural holes between Simmelian ties than in graph d). Our measure of Simmelian brokerage does indeed capture this difference. In fact, if we removed $i$ from graph d), then 10 out of 28 pairs among $i$ 's neighbours (namely all pairs involving the five nodes in the group) would still remain reachable. In this case, the value of Simmelian brokerage of $i$ is $\mathcal{B}_{i}^{d} \simeq$ 6.46. Conversely, the removal of $i$ in e) would produce a more serious damage to the network, since only 4 pairs of neighbours of $i$ over 28 would remain reachable. In this case, the value of Simmelian brokerage is equal to $\mathcal{B}_{i}^{e}=7.0$.

Finally, graph f) is a star, so that by removing node 
$i$ no pair of its neighbours would remain reachable any longer. In this limiting case, the value of local efficiency of node $i$ is equal to zero, and the one of Simmelian brokerage, as well as of effective size, is equal to the node degree, namely $\mathcal{B}_{i}^{f}=8.0$. In general, if two nodes have the same local efficiency, the one having the higher degree has a higher Simmelian brokerage. This is due to the fact that the removal of a high-degree node could potentially leave a higher number of pairs of nodes disconnected, and could therefore cause a more substantial damage to the network than the removal of a node characterised by a relatively low degree.

\section{WEIGHTED GRAPHS: EFFECTIVE STRENGTH AND WEIGHTED SIMMELIAN BROKERAGE}

In this Section, we briefly discuss how the measures of effective size and Simmelian brokerage can be intuitively extended to the case of weighted graphs. First, we notice that, in the more general case of undirected weighted graphs, the importance of node $i$ can be measured through its total strength $s_{i}=\sum_{j} w_{i j}$, in addition to the degree $k_{i}$. Consequently, it is reasonable to extend the measure of effective size to weighed graphs by defining the effective strength of a node as follows:

$$
\begin{aligned}
\mathcal{S}_{i}^{w} & =\sum_{j} w_{i j}\left[1-\sum_{\ell} p_{i \ell} m_{j \ell}\right]= \\
& =s_{i}-\sum_{j} \sum_{\ell} w_{i j} p_{i \ell} m_{j \ell}
\end{aligned}
$$

Like effective size for unweighted graphs, effective strength measures the extent to which the neighbourhood of a node in a weighted graph is redundant. However, unlike effective size, it properly takes into account the weights of links, and thus captures variations in the investment (e.g., time, energy) that a node $i$ makes in each of its neighbours. Since the quantity in square brackets in Equation (17) is multiplied by $w_{i j}$, then a neighbour $j$ of node $i$ 's for which $w_{i j}$ is relatively small has little impact on the effective strength of $i$. Conversely, if $w_{i j}$ is relatively large, then node $j$ can substantially influence the effective strength of $i$. It is easy to verify that, if the graph is unweighted and undirected, the effective strength in Equation (17) reduces to the effective size, as defined in Equation (5).

In principle, starting from Equation (17), it should be possible to derive an exact relation between the effective strength and the weighted clustering coefficient of a node, as we did in Section for the unweighted case, and to extend to weighted graphs the measure of Simmelian brokerage proposed in Equation (16). However, while the definition of clustering coefficient in unweighted graphs reported in Equation (1) is widely accepted and undisputed, there exist more than one manner to define the clustering coefficient of a node in a weighted graph [48]. Indeed, different measures for the weighted clustering coefficient have been proposed in the literature. Among those, the following four are the most popular ones:

$$
\begin{aligned}
& C_{i}^{w}=C_{i}^{B}=\frac{1}{s_{i}\left(k_{i}-1\right)} \sum_{j, \ell} \frac{w_{i j}+w_{i \ell}}{2} a_{i j} a_{j \ell} a_{\ell i} \\
& C_{i}^{w}=C_{i}^{O}=\frac{2 \sum_{j, \ell}\left(w_{i j} w_{j \ell} w_{\ell i}\right)^{1 / 3}}{k_{i}\left(k_{i}-1\right)} \\
& C_{i}^{w}=C_{i}^{Z}=\frac{\sum_{j \neq i} \sum_{j \neq \ell, \ell \neq i}\left(w_{i j} w_{j \ell} w_{\ell i}\right)}{\left(\sum_{j \neq i} w_{i j}\right)^{2}-\sum_{j \neq i} w_{i j}^{2}} \\
& C_{i}^{w}=C_{i}^{H}=\frac{\sum_{j, \ell} w_{i j} w_{j \ell} w_{\ell i}}{\max (w) \sum_{j, \ell} w_{i j} w_{\ell i}}
\end{aligned}
$$

which have been respectively defined by Barrat et al. [3], Onnela et al. [47], Zhang and Horvath [70] and Holme et al. 32]. Notice that all these measures are essentially based on the same idea: the clustering of node $i$ is measured by means of the sum of the weights of the closed triads incident on $i$. Nevertheless, each measure differs from the others in the choice of the weight assigned to each triad and in the normalisation introduced to guarantee that $C_{i}^{w}$ takes values in $[0,1]$. A discussion of the different definitions of the weighted clustering coefficient is beyond the scope of the present paper (the authors of Ref. [56] have carried out a thorough analysis of these measures and a comparison of their properties). In a similar way as in the case of unweighted graphs, for which the relation between effective size, clustering and degree of a node is given by Equation (14), for each of the four definitions of weighted clustering it is possible to find a corresponding functional relation to obtain the effective strength of a node if one knows the value of its weighted clustering coefficient. In general, if we assume that this functional relation is mediated not only by the degree $k_{i}$ of node $i$ but also by the node strength $s_{i}$, we can write:

$$
\mathcal{S}_{i}^{w}=F\left(k_{i}, s_{i}, C_{i}^{w}\right)
$$

where the form of $F\left(k_{i}, s_{i}, C_{i}^{w}\right)$ depends only on the chosen definition of clustering coefficient. Following the same logic described in Section ${ }_{\square}$ we notice that each version of the weighted clustering coefficient induces a different definition of weighted Simmelian brokerage $\mathcal{B}_{i}^{w}$. As we did for the case of unweighted graphs, where the Simmelian brokerage of a node was obtained by substituting $E_{i}$ for $C_{i}$ in Equation (13), we define the weighted Simmelian brokerage of node $i$ induced by a given definition of weighted clustering as follows:

$$
\mathcal{B}_{i}^{w}=F\left(k_{i}, s_{i}, E_{i}^{w}\right)
$$


where $E_{i}^{w}$ is the local efficiency of node $i$ in the weighted graph, which is measured considering the weighted distances $d_{j \ell}^{w}$ instead of the topological distances $d_{j \ell}$. In other words, for a given formulation of weighted clustering, the Simmelian brokerage of a node is obtained by replacing the weighted clustering coefficient $C_{i}^{w}$ with the local efficiency $E_{i}^{w}$ in the function $F\left(k_{i}, s_{i}, C_{i}^{w}\right)$ that relates effective strength to the weighted clustering coefficient.

\section{CONCLUSIONS}

Graphs are an invaluable mathematical tool for examining the topology and evolution of social structures, and graph measures have contributed to the operationalisation and formalisation of fundamental sociological concepts as well as to the development of social theories. Among these measures, clustering and effective size have played a pivotal role in the debate that, over the last few decades, has been concerned with the types of social structures that matter as sources of social capital $[2,44,9,20,23,40,41,54,61]$. In this paper, our contribution to this debate began by reviewing the two measures, clustering and effective size, typically associated with two opposing types of social structure, the closed and open structure respectively. We then clarified the relationship between these two measures, and found that they are indeed connected through a simple mathematical relation. While so far the two measures have been related to each other primarily at a conceptual level and on intuitive grounds [7], in this paper we provided a formal framework in which one measure can be expressed in terms of the other.

The study of formal relations between different graph measures can help unveil the intimate connections between already existing, and apparently unrelated, sociological concepts and, at times, even lead to the development of new concepts and measures. This indeed describes the trajectory that brought us from a more thorough understanding of the relation between closed and open structures to the proposal of a new measure that captures a topological configuration at the interface between the two types of structure. The idea was to identify brokerage positions in which a node can intermediate between otherwise disconnected cohesive groups of contacts [67]. In such cases, the node's local network can be seen as both open and closed: open in that it is rich in structural holes separating distinct groups of contacts; and closed in that it is at the same time rich in third-party relationships within each of the groups with which the node is affiliated.

In qualitative agreement with the organisational literature on Simmelian ties [19, 34 36, 61], we proposed to call Simmelian brokerage the new measure for detecting such structural positions. Simmelian brokerage helps differentiate between brokerage positions of nodes with the same degree and the same local clustering coefficient, but with a different configuration of links in their local neighbourhoods. In those cases, effective size would also remain unchanged as, all else being equal, it is not sensitive to variations in the positions of links. However, brokerage opportunities are likely to differ when, simply by reshuffling the same number of links across a node's local neighbourhood, there is a variation in the number of socially cohesive groups with which the node is affiliated. Simmelian brokerage, unlike effective size, is sensitive precisely to these variations in group affiliation that result from a change in the position of links.

Our findings can nourish the theoretical debate over the relative salience of closed and open structures for social capital, and will inform further research on the generative mechanisms of social capital. On the one hand, empirical tests of the relative benefits of closed and open structures will now find in our proposed relation between clustering and effective size a sound argument safeguarding against problems of multicollinearity, typically arising as a result of the simultaneous inclusion of both measures as explanatory variables in multivariate regression models. On the other, future research on the relative benefits of cohesive and brokered networks will benefit from the application of Simmelian brokerage to a number of empirical domains. In this sense, our study will help reconcile the apparently opposing results that various strands of literature have uncovered on the structural foundations of social capital [1, 2, 20, 23, 33, 42, 53].

Simmelian brokerage, as a new topological measure of network structure, can also spur a wealth of research broadly concerned with the topology and dynamics of complex networks. For the sake of simplicity, in this paper we have restricted our focus primarily to the case of unweighted networks. However, as was sketched out in Section . the relation between clustering coefficient and effective size can easily be generalised to the case of weighted graphs, which will in turn enable Simmelian brokerage to be also extended to weighted graphs.

More generally, the main implication of our study for research on complex networked systems lies in the change of perspective entailed by our emphasis on structural cleavages, as opposed to ties, that we borrowed from the burgeoning network literature in the social sciences. In this sense, our study may suggest a number of possible and previously neglected ways in which, simply by deflecting attention from the presence to the absence of a tie, new insights can be gained on the organisation, functioning and dynamics of a variety of systems.

[1] Ahuja, G.: Collaboration networks, structural holes, and innovation: A longitudinal study. Administrative Science 
Quarterly. 45, 425-455 (2000).

[2] Aral, S., Van Alstyne, M.: The diversity-bandwidth trade-off. American Journal of Sociology. 117(1), 90-171 (2011).

[3] Barrat, A., Barthélemy, M., Pastor-Satorras, R. and Vespignani, A.: The architecture of complex weighted networks. Proceedings of the National Academy of Sciences of the United States of America. 101, 3747-3752 (2004).

[4] Baum, J.A.C., McEvily, B., Rowley, T.J.: Better with age? Tie longevity and the performance Implications of bridging and closure. Organization Science. 23, 529-546 (2012).

[5] Borgatti, S.P.: Structural holes: Unpacking Burt's redundancy measures. Connections. 20, 35 (1997).

[6] Brass, D.J.: It's all in your social network. In: Ford, C.M., Gioia, D.A. (eds.), Creative Action in Organizations, pp. 94-98. Sage, Thousand Oaks, CA (1995).

[7] Burt, R.S.: Structural Holes. The Social Structure of Competition. Harvard University Press, Cambridge MA (1992).

[8] Burt, R.S.: Structural holes and good ideas. American Journal of Sociology. 110, 349-399 (2004).

[9] Burt, R.S.: Brokerage and Closure. Oxford University Press, Oxford (2005).

[10] Burt, R.S.: Neighbor Networks. Oxford University Press, Oxford (2010).

[11] Burt, R. S.: The gender of social capital. Rationality and Society. 10, 5-46 (1998).

[12] Burt, R. S., and Knez, M.: Kinds of third-party effects on trust. Rationality and Society. 7, 255-292 (1995).

[13] Centola, D., Macy, M.W.: Complex contagion and the weakness of long ties. American Journal of Sociology. 113, 702-734 (2007).

[14] Coleman, J. S.: Social capital in the creation of human capital. American Journal of Sociology. 94, S95-S120 (1988).

[15] Coleman, J.S.: Foundations of Social Theory. Harvard University Press, Cambridge, MA (1990).

[16] Cover T.M., Thomas J. A.: Elements of Information Theory. Wiley, New York (1991).

[17] Davis, J.A.: Clustering and hierarchy in interpersonal relations: Testing two graph theoretical models on 742 sociomatrices. American Sociological Review. 35(5), 843851 (1970).

[18] Davis, J.A., Holland, P.W., Leinhardt, S.: Comments on Professor Mazur's hypothesis about interpersonal sentiments. American Sociological Review. 36, 309-311 (1971).

[19] Dekker, D.: Measures of Simmelian tie strength, Simmelian brokerage, and Simmelianly brokered. Journal of Social Structure. 7(1), 1-22 (2006).

[20] Fleming, L., Mingo, S., Chen, D.: Collaborative brokerage, generative creativity, and creative success. Administrative Science Quarterly. 52, 443-475 (2007).

[21] Fortunato, S.: Community detection in graphs. Physics Reports. 486, 75-174 (2010).

[22] Friedkin, N.E.: Social Cohesion. Annual RevIew of Sociology. 30, 409-25 (2004).

[23] Gargiulo, M., Benassi, M.: Trapped in your own net? Network cohesion, structural holes, and the adaptation of social capital. Organization Science. 11: 183-196 (2000).

[24] Gargiulo, M., Ertug, G., Galunic, C.: The two faces of control: Network closure and individual performance among knowledge workers. Administrative Science
Quarterly. 54, 299-333 (2009).

[25] Gould, R.V.: Multiple networks and mobilization in the Paris Commune, 1871. American Sociological Review. 56, 716-729 (1991).

[26] Granovetter, M.: The strength of weak ties. American Journal of Sociology. 78, 1360-1380 (1973).

[27] Granovetter, M.: The impact of social structure on economic outcomes. Journal of Economic Perspectives. 19(1), 33-50 (2005).

[28] Hansen, M.T.: The search-transfer problem: The role of weak ties in sharing knowledge across organization subunits. Administrative Science Quarterly. 44, 232-248 (1999).

[29] Holland, P.W., Leinhardt, S.: A method for detecting structure in sociometric data. American Journal of Sociology. 76, 492-513 (1970).

[30] Holland, P.W., Leinhardt, S.: Transitivity in structural models of small groups. Comparative Group Studies. 2, 107-124 (1971).

[31] Holme, P. Kim, B. J.: Growing scale-free networks with tunable clustering. Phys. Rev. E. 65, 026107 (2002).

[32] Holme, P., Park, S. M., Kim, B. J. and Edling, C. R.: Korean university life in a network perspective: Dynamics of a large affiliation network. Physica A: Statistical Mechanics and its Applications. 373, 821-830 (2007).

[33] Ingram, P., Roberts, P.W.: Friendships among competitors in the Sydney hotel industry. American Journal of Sociology. 106(2), 387-423 (2000).

[34] Krackhardt, D.: Simmelian tie: Super strong and sticky. In: R. M. Kramer, R.M., Neale, M.A. (eds.), Power and influence in organizations, p. 21-38. Sage, Thousand Oaks, CA (1998).

[35] Krackhardt, D.: The ties that torture: Simmelian tie analysis in organizations. Research in the Sociology of Organizations. 16, 183-210 (1999).

[36] Krackhardt, D., Kilduff, M.: Structure, culture and Simmelian ties in entrepreneurial firms. Social Networks. 24, 279-290 (2002).

[37] Lambiotte, R., Panzarasa, P.: Communities, knowledge creation, and information diffusion. Journal of Informetrics. 3(3), 180-190 (2009).

[38] Latora, V., Marchiori, M.: Efficient behavior of smallworld networks. Phys. Rev. Lett. 87, 198701 (2001).

[39] Latora, V., Marchiori, M.: Economic behavior of smallworld networks. Eur. Phys. J. B. 32, 249-263 (2003).

[40] Lin, N.: Social Capital. A Theory of Social Structure and Action. Cambridge University Press, New York (2001).

[41] Lin, N., Cook, K., Burt, R.S. (eds.): Social Capital. Theory and Research. Aldine Transaction, New Brunswick and London (2001).

[42] Long Lingo, E., O'Mahony, S.: Nexus work: Brokerage on creative projects. Administrative Science Quarterly. 55, 47-81 (2010).

[43] Luce, R.D., Perry, A.D.: A method of matrix analysis of group structure. Psychometrika. 14(1), 95-116 (1949).

[44] Mizruchi, M., Stearns, L.B.: Getting deals done: The use of social networks in bank decision-making. American Sociological Review. 66, 647- 471 (2001).

[45] Nahapiet, J., Ghoshal, S.: Social capital, intellectual capital, and the organizational advantage. Academy of Management Review. 23, 242-266 (1998).

[46] Obstfeld, D.: Social networks, the tertius iungens orientation, and involvement in innovation. Administrative Science Quarterly. 50, 100-130 (2005). 
[47] Onnela, J.-P., Saramäki, J., Kertész, J. and Kaski, K.: Intensity and coherence of motifs in weighted complex networks. Phys. Rev. E. 71, 065103 (2005).

[48] Opsahl, T., Panzarasa, P.: Clustering in weighted networks. Social Networks. 31, 155-163 (2009).

[49] Palla, G., Derényi, I., Farkas, I., Vicsek. T.: Uncovering the overlapping community structure of complex networks in nature and society. Nature. 435 (7043), 814-818 (2005).

[50] Perry-Smith, J.E.: Social yet creative: The role of social relationships in facilitating individual creativity. Academy of Management Journal. 49, 85-101 (2006).

[51] Podolny, J. M., Baron, J. N.: Resources and relationships: Social networks and mobility in the workplace. American Sociological Review. 62, 673-693 (1997).

[52] Ravasz, E., Barabási, A.-L.: Hierarchical organization in complex networks. Phys. Rev. E 67, 026112 (2003).

[53] Reagans, R., McEvily, B.: Network structure and knowledge transfer: The effects of cohesion and range. Administrative Science Quarterly. 48, 240-267 (2003).

[54] Reagans, R., Zuckerman, E.: Networks, diversity and performance: The social capital of R\&D units. Organization Science. 12, 502-517 (2001).

[55] Rodan, S., Galunic, C.: More than network structure: How knowledge heterogeneity influences managerial performance and innovativeness. Strategic Management Journal, 25, 541-562 (2004).

[56] Saramäki, J., Kivelä, M., Onnela, J.-P., Kaski, K. and Kertész, J.: Generalizations of the clustering coefficient to weighted complex networks. Phys. Rev. E. 75, 027105 (2007).

[57] Serrano, M.A., Boguñá, M.: Tuning clustering in random networks with arbitrary degree distributions. Phys. Rev. E. 72, 036133 (2005).

[58] Simmel, G.: The Sociology of Georg Simmel. Trans. by K.H. Wolff. Lightning Source, Milton Keynes UK [1923] (2011).

[59] Sosa, M.E.: Where do creative interactions come from?
The role of tie content and social networks. Organization Science. 22, 1-21 (2011).

[60] Stovel, K., Shaw, L.: Brokerage. Annual Review of Sociology. 38, 139-158 (2012).

[61] Tortoriello, M., Krackhardt, D.: Activating crossboundary knowledge: The role of Simmelian ties in the generation of innovations. Academy of Management Journal. 53(1), 167-181 (2010).

[62] Tortoriello, M., Reagans, R., McEvily, B.: Bridging the knowledge gap: The influence of strong ties, network cohesion, and network range on the transfer of knowledge between organizational units. Organization Science. 23, 1024-1039 (2012).

[63] Uzzi, B.: Social structure and competition in interfirm networks: The paradox of embeddedness. Administrative Science Quarterly. 43, 35-67 (1997).

[64] Uzzi, B., Spiro, J.: Collaboration and creativity: The small world problem. American Journal of Sociology. 111, 447-504 (2005).

[65] Vázquez, A., Pastor-Satorras, R., Vespignani, A.: Largescale topological and dynamical properties of the Internet. Phys. Rev. E. 65, 066130 (2002).

[66] Vázquez, A.: Growing network with local rules: Preferential attachment, clustering hierarchy, and degree correlations. Phys. Rev. E. 67, 056104 (2003).

[67] Vedres, B., Stark, D.: Structural folds: Generative disruption in overlapping groups. American Journal of Sociology. 115(4), 1150-1190 (2010).

[68] Watts, D.J.: Small Worlds: The Dynamics of Networks between Order and Randomness. Princeton University Press, Princeton, New Jersey (1999).

[69] Watts, D.J., Strogatz, S.H.: Collective dynamics of small-world networks. Nature. 393, 440 (1998).

[70] Zhang, B., Horvath, S.: A general framework for weighted gene co-expression network analysis. Stat. App. Genet. Mol. Biol. 4, 17 (2005). 